\title{
CARACTERÍSTICAS CLÍNICAS DE HOMENS ATENDIDOS EM UNIDADE DE PRONTO ATENDIMENTO
}

\author{
CLINICAL CHARACTERISTICS OF MEN MET \\ IN EMERGENCY CARE UNIT
}

\section{CARACTERÍSTICAS CLÍNICAS DE LOS HOMBRES ATENDIDOS EN LA UNIDAD DE CUIDADOS DE EMERGENCIA}

\author{
Jéssica Cerqueira Silva ${ }^{1}$ \\ Silvia de Sousa Nassif ${ }^{2}$ \\ Anderson Reis Sousa ${ }^{3}$ \\ Selton Diniz dos Santos ${ }^{4}$ \\ Tilson Nunes Mota \\ Álvaro Pereira ${ }^{6}$
}

Como citar este artigo: Silva JC, Nasif SS, Sousa AR, Santos SD, Mota TN, Pereira A. Características clínicas de homens atendidos em unidade de pronto atendimento. Rev baiana enferm. 2020;34:e34702.

Objetivo: descrever o perfil clínico de homens atendidos em uma unidade de pronto atendimento, do setor público de saúde, em uma cidade do Nordeste brasileiro. Método: estudo descritivo, realizado mediante consulta a 500 prontuários de atendimento de homens em unidade de emergência, no período de janeiro a dezembro de 2015. Resultados: a queixa principal foi dor (35,2\%), situação clínica estável $(90,4 \%)$, classificação de risco não urgente (47,9\%), sem apresentação de agravos pré-existentes (80,2\%), diagnóstico médico dor em geral (22,6\%), terapêutica instituída foi a medicamentosa $(87,4 \%)$, evolução do caso foi alta por melhora $(91,6 \%)$, não houve necessidade de encaminhamento $(88,0 \%)$ e não houve registros de diagnósticos de enfermagem $(96,8 \%)$. Conclusão: o perfil clínico de homens atendidos em uma unidade de pronto atendimento no Nordeste brasileiro apresenta como características situações de baixa complexidade sem alterações do nível de estabilidade hemodinâmica e necessidade de outros recursos diagnósticos e terapêuticos.

Descritores: Saúde do Homem. Perfil da Saúde. Serviços Médicos de Urgência. Assistência Ambulatorial.

Objective: to describe the clinical profile of men met in an emergency care unit, of the public bealth sector, in a city in Northeastern Brazil. Method: descriptive study, conducted through analysis of 500 records of men met in the emergency unit, in the period from January to December 2015. Results: the main complaint was pain (35.2\%), stable clinical situation (90.4\%), non-urgent risk classification (47.9\%), without pre-existing injuries (80.2\%), medical diagnosis of pain in general (22.6\%), the therapy used was medication (87.4\%), the case evolution was recovery-related discharge (91.6\%), there was no need for referral (88.0\%) and there were no records of nursing diagnoses (96.8\%). Conclusion: the clinical profile of men met in a bealth care unit in Northeastern Brazil presents

\footnotetext{
Enfermeira. Pesquisadora Independente. Feira de Santana, Bahia, Brasil. https://orcid.org/0000-0002-99| I-6278.

Enfermeira. Pesquisadora independente. Feira de Santana, Bahia, Brasil. https://orcid.org/0000-0002-23 I 9-5627.

Enfermeiro. Mestre em Enfermagem. Professor Assistente da Universidade Federal da Bahia. Salvador, Bahia, Brasil. son.reis@hotmail.com https://orcid.org/0000$0001-8534-1960$

4 Enfermeiro. Mestre em Saúde Coletiva. Professor Auxiliar da Universidade Estadual de Feira de Santana. Feira de Santana, Bahia, Brasil. https://orcid.org/0000-00023992-4353.

5 Enfermeiro. Mestre em Enfermagem. Doutorando Universidade Federal da Bahia, Salvador, Bahia, Brasil. https://orcid.org/0000-000 I-5836-2360.

6 Enfermeiro. Doutor em Enfermagem. Professor Titular da Universidade Federal da Bahia. Salvador, Bahia, Brasil. https://orcid.org/0000-0003-16I5-5528.
} 
as characteristics situations of low complexity without changes in the level of hemodynamic stability and the need for other diagnostic and therapeutic resources.

Descriptors: Men's Health. Health Profile. Urgency Medical Services. Ambulatory Care.

Objetivo: describir el perfil clinico de hombres atendidos en una unidad de cuidados de emergencia, del sector público de salud, en una ciudad en el noreste de Brasil. Método: estudio descriptivo, consultando 500 registros de atendimiento de los hombres en la unidad de emergencia, en el periodo de enero a diciembre de 2015. Resultados: la queja principal fue el dolor (35,2\%), situación clinica estable (90,4\%), clasificación de riesgo no urgente (47,9\%), sin lesiones preexistentes (80,2\%), diagnóstico médico dolor en general (22,6\%), el tratamiento fue medicamentoso (87,4\%), la evolución del caso fue alta por mejora (91,6\%), no bubo necesidad de encaminamiento (88,0\%) y no hubo registros de diagnósticos de enfermería (96,8\%). Conclusión: el perfil clínico de hombres atendidos en una unidad de cuidados de salud en el nordeste brasileño presenta como características situaciones de baja complejidad sin cambios en el nivel de estabilidad hemodinámica y la necesidad de otros recursos diagnósticos y terapéuticos.

Descriptores: Salud del Hombre. Perfil de Salud. Servicios Médicos de Emergencia. Atención Ambulatoria.

\section{Introdução}

As estratégias e ações voltadas para a saúde de homens é uma prioridade que emergiu nas políticas públicas de saúde no Brasil a partir do ano de 2009, após a implantação da Política Nacional de Atenção Integral à Saúde do Homem $(\mathrm{PNAISH})^{(1)}$. O atual panorama epidemiológico brasileiro tem mostrado um perfil clínico de saúde que aponta para a elevada morbimortalidade masculina ${ }^{(2-3)}$. Tal cenário revela que as mortes de homens têm sido precoces e relacionam-se com a manutenção e a prática de comportamentos de risco, expressos pelo consumo abusivo de álcool e outras drogas, violências, acidentes de trabalho e transtornos mentais ${ }^{(4-5)}$, assim como à adoção de hábitos não saudáveis que repercutem no elevado crescimento das comorbidades crônicas.

Além desse comportamento de risco, outros aspectos mostram-se notórios na relação existente entre homens e cuidados com a saúde. Nesse âmbito, operam, com grande potencial de influência, as construções sociais das masculinidades hegemônicas. Estas favorecem a percepção de invulnerabilidade, força, virilidade e honra, que distanciam os homens da lógica do estabelecimento de um comportamento cuidativo, impactando na busca e no pertencimento em relação aos serviços de saúde, em especial os da Atenção Primária ${ }^{(6-7)}$.

$\mathrm{Na}$ busca por soluções de questões relacionadas às demandas clínicas de saúde, os homens saltam o modelo de atenção, frequentando, em maior expressividade, as unidades de urgência e emergência, a exemplo das Unidades de Pronto Atendimento 24 horas (UPA) ${ }^{(8)}$. Alguns fatores podem estar relacionados com a elevada procura dos homens pelos serviços de caráter emergencial na saúde, a exemplo de: descaracterização da Atenção Básica, ausência da criação de estratégias de sensibilização e captação masculina, dificuldade de acesso às consultas médicas e outros recursos terapêuticos na Atenção Básica, incompatibilidade de horários entre o trabalho e o funcionamento dos serviços de saúde na Atenção Básica, cultura do prolongamento de sintomas, assim como o imaginário social de que as unidades de urgência e emergência são mais rápidas e resolutivas ${ }^{(9-10)}$.

Por se caracterizarem serviços de porta aberta 24 horas, as UPA compõem a Rede de Atenção às Urgências no Brasil e são responsáveis por atender grande parte das demandas de saúde apresentadas pelos usuários nos territórios. Essas demandas incluem desde aquelas que são de responsabilidade e alcance dessas unidades até aquelas que extrapolam os níveis de recursos e atenção a serem dispensados ou ainda aquelas cujas características não correspondem às ocorrências de urgência e emergência, mas estão presentes e em expressivo volume numérico ${ }^{(11)}$.

Como exemplo da consequência da busca de homens por demandas de menor complexidade 
clínica, cita-se a apresentação de alterações mínimas dos sinais vitais, com preservação do nível de consciência e em presença de estabilidade hemodinâmica, que poderia ser solucionada nas Unidades de Saúde da Família (USF) e Unidades Básicas de Saúde (UBS). Por consequência, são evidenciados cenários de superlotação dos serviços de urgência e emergência, impactos no processo de trabalho em saúde, sobrecarga dos profissionais e trabalhadores, dificuldade de organização dos serviços e da provisão de recursos humanos e materiais, inadequação estrutural em comparação com a característica da demanda apresentada, desarticulação com a rede de Atenção Primária e comprometimentos da qualidade da assistência e da segurança do paciente ${ }^{(11-12)}$. Tal problemática exige que as UPA sejam integradas a uma política de redes de atenção à saúde, como forma de garantir resolubilidade por meio da ampliação de seus serviços ${ }^{(13)}$.

Estudo realizado em grandes cidades belgas sobre o perfil do atendimento fora do horário comercial, realizado por pacientes atendidos no pronto socorro e por clínicos gerais de plantão, apontou elevada procura, com fatores determinantes associados. Esses fatores estão relacionados a ser homem, ter feito uso excessivo de serviços de emergência nos últimos 12 meses, ter nacionalidade africana e não possuir plano ou seguro de saúde. O estudo ainda sugere que a procura elevada, e por vezes considerada inapropriada, pelos serviços de emergência configura-se em uma problemática preocupante, por comprometer o uso de pessoal, infraestrutura e recursos financeiros dos serviços, necessitando de redirecionamento dos pacientes, por meio do conhecimento ampliado acerca das experiências, atitudes e comportamento de escolha desses ${ }^{(14)}$.

Embora previsto na Política Nacional de Redução da Morbimortalidade por Acidentes e Violência implantada no Brasil em 2001, a sistematização de informações epidemiológicas sobre o perfil dos atendimentos em unidades de Pronto Socorros e Pronto Atendimentos é deficitária, comprometendo a compreensão de indicadores e das condições sensíveis para $\mathrm{O}$ desenvolvimento de ações voltadas à gestão do cuidado e a atenção à saúde na rede de urgências $^{(15)}$. Em contrapartida, a Pesquisa Nacional de Saúde revelou, sobre o acesso e utilização dos serviços de saúde no Brasil, que a UBS foi o estabelecimento mais procurado pela população, quando necessitou de atendimento de saúde. Todavia, quando incluídos as unidades de Pronto Atendimento ou emergências de hospitais públicos e privados, assim como os hospitais públicos ou ambulatórios, as policlínicas e os Postos de Assistência Médica, o número da busca torna-se expressivo ${ }^{(16)}$.

A pesquisa referida revelou ainda que a procura de homens por atendimento de saúde foi motivada mais frequentemente pela presença de doença, seguida de continuidade de tratamento e realização de exame complementar e diagnóstico. A continuidade dos tratamentos ocorreu, em maior número, entre pessoas com idade entre 40 e 59 anos. Quando observada a procura de atendimento de saúde decorrente de acidentes ou lesões, os homens ocupavam a maior posição, quando comparados com as mulheres ${ }^{(16)}$.

No que tange às demandas apresentadas pelos usuários nas unidades de emergência, verificadas no sistema de Acolhimento com Classificação de Risco, o perfil é caracterizado por demandas de menor nível de urgência, o que sinaliza, portanto, menor grau de priorização no atendimento $^{(8)}$. Entre as razões que conduzem os homens aos serviços de emergência, a pesquisa prospectiva realizada na Austrália sobre as diferenças de gênero entre mulheres e homens que acessaram uma unidade de pronto socorro evidenciou a prevalência masculina elevada para os agravos cardiovasculares, com chances mais elevadas de terem que se submeter a uma angiografia e revascularização miocárdica do que o público feminino ${ }^{(17)}$.

Estudo realizado em uma emergência hospitalar de um hospital de Belo Horizonte, Minas Gerais, Brasil, ao observar o levantamento de Diagnósticos de Enfermagem apresentados por pacientes em unidade de emergência, maior prevalência de nutrição desequilibrada - menos 
do que as necessidades corporais -, identificou risco de integridade da pele prejudicada, risco de infecção, padrão respiratório ineficaz e ventilação espontânea prejudicada ${ }^{(18)}$. Diante desse cenário, reorganizar a demanda de atendimento, bem como redirecionar a procura dos serviços de saúde por parte do público masculino, tem se configurado um desafio contemporâneo relevante a ser superado, tanto por parte dos promulgadores de políticas públicas quanto dos profissionais e trabalhadores da saúde ${ }^{(11-13)}$.

O conhecimento das demandas de saúde da população masculina poderá subsidiar o planejamento de ações específicas direcionadas às suas necessidades, ampliando o potencial de ação dos serviços de saúde. Além disso, fornecerá informações que consubstanciem a sensibilização de homens para a busca dos serviços de saúde do Sistema Único de Saúde (SUS) que sejam ideais para a resolução de seus problemas, de acordo com o grau de complexidade de atenção.

Frente ao exposto, com a intenção de fomentar o levantamento de evidências científicas que auxiliem a prática profissional em saúde voltada para a atenção masculina, buscou-se investigar: Como se configura o perfil clínico de homens atendidos em uma unidade de pronto atendimento?

Este estudo tem como objetivo descrever o perfil clínico de homens atendidos em uma unidade de pronto atendimento do setor público de saúde em uma cidade do Nordeste brasileiro.

\section{Método}

Estudo descritivo, documental, realizado em uma Unidade de Pronto Atendimento de 24 horas do município de Feira de Santana (BA), sobre o perfil clínico de atendimento de homens no ano de 2015.

Foram critérios de inclusão: homens adultos e idosos, com idade maior que 20 anos, conforme estabelecido pela PNAISH, atendimento datado de janeiro a dezembro de 2015 e disponibilidade de prontuário de atendimento na unidade. Foram excluídos homens que não tiveram avaliação médica e/ou aqueles com mais de uma variável avaliada faltante.

Para o cálculo amostral foi considerada a população de 34.669 homens atendidos (segundo registro da unidade de pronto atendimento onde o estudo foi realizado), prevalência de 59,3\% para o atendimento de homens nesse tipo de serviço ${ }^{(19)}$, precisão de erro de 5\%, nível de confiança de $95 \%$, poder de $80 \%$ e adicional de perda de 20\%. A amostra mínima foi definida em 442, tendo sido analisados 500 prontuários. Estes foram selecionados por meio de amostragem aleatória sistemática, considerando-se o intervalo amostral igual a 79. Os prontuários foram organizados por numeração sequencial de admissão. Realizou-se substituição dos prontuários excluídos por aquele diretamente posterior ao intervalo amostral. Quando este não foi incluído, considerou-se o anterior ao intervalo.

A coleta de dados foi realizada nos meses de outubro e novembro de 2016. Utilizou-se um formulário estruturado como instrumento para coleta dos dados, constituído por variáveis referentes às características sociodemográficas (faixa etária, raça/cor, estado civil) e clínicas (queixa principal, situação clínica, classificação de risco, agravos preexistentes, diagnóstico médico, terapêutica, evolução do caso, tipo de encaminhamento e evolução de enfermagem).

Utilizou-se o software Statistic Package Science for Social (SPSS), versão 23.0, para processamento e tratamento dos dados organizados previamente em planilha do programa Microsoft Excel 2010. Realizou-se análise descritiva dos dados, estimando-se frequências absolutas e relativas, com apresentação em tabelas.

Em atendimento à Resolução n. 466, de 12 de dezembro de 2012, do Conselho Nacional de Saúde, o estudo foi aprovado pelo Comitê de Ética em Pesquisa da Faculdade Anísio Teixeira, Parecer n. 1.748.594.

\section{Resultados}

Dos 500 homens atendidos no pronto atendimento, observou-se maior frequência da faixa 
etária de 30 a 39 anos, com identificação ignorada da raça/cor, estado civil, escolaridade, situação laboral e renda.

Sobre as características clínicas, predominaram: dor como queixa principal, condições clínicas não urgentes, situação clínica estável, classificação de risco não urgente, sem agravos preexistentes. Entre os preexistentes, as doenças do aparelho circulatório, diagnóstico médico de dor em geral, terapêutica medicamentosa, evolução do caso de alta por melhora, nenhum encaminhamento do caso e ausência de registro de Diagnósticos de Enfermagem, foram os mais recorrentes (Tabela 1 ).

Tabela 1 - Características clínicas do atendimento de homens em uma unidade de urgência/emergência.

Feira de Santana, Bahia, Brasil - 2015 (N=500)

\begin{tabular}{|c|c|c|}
\hline Variáveis & $\mathbf{n}$ & $\%$ \\
\hline \multicolumn{3}{|l|}{ Queixa principal/motivo do atendimento $(n=500)$} \\
\hline Dor & 179 & 35,8 \\
\hline Dispneia & 24 & 4,8 \\
\hline Acidente motociclístico & 27 & 5,4 \\
\hline Paciente psiquiátrico & 6 & 1,2 \\
\hline Amigdalite & 16 & 3,2 \\
\hline Queda & 14 & 2,8 \\
\hline Precordialgia & 17 & 3,4 \\
\hline Consumo excessivo de bebida alcoólica & 6 & 1,2 \\
\hline Mal-estar & 14 & 2,8 \\
\hline Diabetes & 11 & 2,2 \\
\hline Tontura e náuseas & 9 & 1,8 \\
\hline Acidente por arma branca & 8 & 1,6 \\
\hline Febre & 28 & 5,6 \\
\hline Epigastralgia & 26 & 5,2 \\
\hline HAS & 21 & 4,2 \\
\hline Condições clínicas não urgentes & 94 & 18,8 \\
\hline \multicolumn{3}{|l|}{ Situação clínica $(n=500)$} \\
\hline Estável & 452 & 90,4 \\
\hline Instável & 48 & 9,6 \\
\hline \multicolumn{3}{|l|}{ Classificação de risco $(n=499)$} \\
\hline Emergência & 36 & 7,2 \\
\hline Urgência & 224 & 44,9 \\
\hline Não Urgência & 239 & 47,9 \\
\hline \multicolumn{3}{|l|}{ Agravos preexistentes $(n=499)$} \\
\hline Doenças do aparelho circulatório & 62 & 12,4 \\
\hline Doenças metabólicas & 10 & 2,0 \\
\hline Doenças sexualmente transmissíveis & 2 & 0,4 \\
\hline Doenças infecciosas & 7 & 1,4 \\
\hline Doenças do aparelho digestivo & 1 & 0,2 \\
\hline Desconforto respiratório & 2 & 0,4 \\
\hline $\begin{array}{l}\text { Doenças Crônicas associadas (Hipertensão Arterial } \\
\text { Sistêmica+Diabetes Mellitus) }\end{array}$ & 10 & 2,0 \\
\hline Outros $^{(1)}$ & 5 & 1,0 \\
\hline Sem agravos & 400 & 80,2 \\
\hline \multicolumn{3}{|l|}{ Diagnóstico médico (n=473) } \\
\hline Dor em geral & 107 & 22,6 \\
\hline Doenças do aparelho circulatório & 60 & 12,7 \\
\hline Sintomas, sinais e afecções mal definidos & 51 & 10,8 \\
\hline Lesões, envenenamentos e causas externas & 47 & 9,9 \\
\hline Doenças osteomusculares e do tecido conjuntivo & 43 & 9,1 \\
\hline Doenças do aparelho respiratório & 41 & 8,7 \\
\hline
\end{tabular}


Tabela 1 - Características clínicas do atendimento de homens em uma unidade de urgência/emergência.

Feira de Santana, Bahia, Brasil - 2015 (N=500)

(conclusão)

\begin{tabular}{|c|c|c|}
\hline Variáveis & $\mathbf{n}$ & $\%$ \\
\hline \multicolumn{3}{|l|}{ Diagnóstico médico $(n=473)$} \\
\hline Doenças do sistema nervoso & 38 & 8,0 \\
\hline Doenças infecciosas e parasitárias & 35 & 7,4 \\
\hline Doenças do aparelho digestivo & 10 & 2,1 \\
\hline Doenças metabólicas & 8 & 1,7 \\
\hline Doenças mentais & 7 & 1,5 \\
\hline Olhos e anexos, ouvidos e apófise mastoide. & 7 & 1,5 \\
\hline Doenças do aparelho urinário & 5 & 1,1 \\
\hline $\begin{array}{l}\text { Doenças do sangue e dos órgãos hematopoiéticos e alguns } \\
\text { transtornos imunitários }\end{array}$ & 1 & 0,2 \\
\hline Outros $(2)$ & 13 & 2,7 \\
\hline \multicolumn{3}{|l|}{ Terapêutica $(n=500)$} \\
\hline Medicamentosa & 437 & 87,4 \\
\hline Cirúrgica & 3 & 0,6 \\
\hline Raios-X & 1 & 0,2 \\
\hline Imobilização & 6 & 1,2 \\
\hline Sutura & 14 & 2,9 \\
\hline Controle de Sinais Vitais (SSVV) & 2 & 0,4 \\
\hline Troca de Sonda Vesical & 1 & 0,2 \\
\hline Desbridamento & 1 & 0,2 \\
\hline Abcesso & 1 & 0,2 \\
\hline Sem intervenções & 34 & 6,7 \\
\hline \multicolumn{3}{|l|}{ Evolução do caso $(n=488)$} \\
\hline Alta por melhora & 447 & 91,6 \\
\hline Transferência por agravamento & 25 & 5,1 \\
\hline Referência para Atenção Básica à Saúde & 15 & 3,1 \\
\hline Óbito & 1 & 0,2 \\
\hline \multicolumn{3}{|l|}{ Tipo de encaminhamento $(n=500)$} \\
\hline Hospital de referência & 25 & 5,0 \\
\hline Programa Saúde da Família & 9 & 1,8 \\
\hline Centro de Atenção Psicossocial & 6 & 1,2 \\
\hline Cardiologista & 4 & 0,8 \\
\hline Ortopedista & 8 & 1,6 \\
\hline Outros $^{(3)}$ & 8 & 1,6 \\
\hline Não encaminhado & 440 & 88,0 \\
\hline \multicolumn{3}{|l|}{ Diagnósticos de Enfermagem $(n=500)$} \\
\hline Encontrado & 16 & 3,2 \\
\hline Não encontrado & 484 & 96,8 \\
\hline Total & 500 & $100 \%$ \\
\hline
\end{tabular}

Fonte: Elaboração própria.

(1) Cisto renal, fratura de fêmur, crise convulsiva, tecido necrosado e prurido.

(2) Hérnia de disco, obstrução de sonda vesical, etilismo crônico, icterícia, virose.

(3) Centro de Saúde Especializado Municipal, angiologista, otorrino, Raio-X de crânio, dentista e urologista.

\section{Discussão}

Ao descrever-se o perfil clínico de atendimento direcionado aos homens em unidades de emergência, devem ser considerados, dentre outros, fatores como idade, escolaridade, situação clínica, motivo de atendimento, diagnóstico médico e classificação de risco, que se inter-relacionam e exercem influências na utilização de serviços de saúde por esse grupo populacional. 
Essa influência é também permeada por questões, como necessidade e disponibilidade de serviços de saúde, propensão da população em utilizá-los e facilidade de acesso ${ }^{(20)}$. Portanto, quando são analisados os perfis de atendimento, torna-se essencial considerar os múltiplos elementos presentes, para fornecer subsídios que possibilitem o acompanhamento da implementação de políticas públicas de saúde.

Os homens na faixa etária adulta jovem, que frequentam os serviços de urgência, necessitam de ações de saúde direcionadas, como alternativa para a diminuição da morbimortalidade por causas externas e condições graves de saúde. Além disso, precisam do fortalecimento da implementação da PNAISH, para que haja efetiva redução dos custos no setor saúde, em decorrência de complicações severas, bem como da redução da população economicamente ativa ${ }^{(21)}$.

Em se tratando da utilização dos serviços de média complexidade à saúde pelo público masculino, observa-se que é motivada pela apresentação de sinais e sintomas de agravos já instalados, conforme indica estudo nacional ${ }^{(22)}$. Observou-se que a maior parte do público masculino atendido referiu não ter agravos pré-existentes. Aqueles que os referiram, tinham agravos cardiovasculares. Comumente, os próprios homens adultos têm referido utilizar com maior frequência os serviços de saúde disponíveis na rede de urgência e emergência, para a resolução dos seus problemas de saúde. Esta procura tem sido justificada pela rapidez, agilidade no acesso e atendimento às demandas ${ }^{(22)}$.

Nesses cenários, o público masculino atendido nos serviços de urgência apresenta sensação dolorosa classificada, na maior parte dos casos, de moderada a intensa, em especial nos casos de agravos ortopédicos ${ }^{(23)}$. Apesar da dor intensa, as condições clínicas comumente apresentadas por eles nesses serviços não são urgentes, e as condições de saúde que apresentam são estáveis e implicam em terapêuticas de menor complexidade. Destarte, o cumprimento da prescrição medicamentosa e a adoção de condutas médicas desarticuladas da rede de atenção expressam-se na ausência de encaminhamentos para os demais serviços de saúde. Desse modo, essas demandas, que poderiam ter sido resolvidas na Atenção Básica à Saúde, geram uma superlotação nos serviços.

Entre as principais terapêuticas instituídas na UPA, constatou-se que os homens foram submetidos à administração de medicamentos, seguida de suturas e imobilizações. Tais terapêuticas encontram-se relacionadas às queixas apresentadas e aos diagnósticos médicos levantados, para garantir resposta à dor, bem como à presença de lesões decorrentes de causas externas. No entanto, evidenciou-se número pouco expressivo de avaliações mediadas por diagnósticos por imagem, tal como de avaliação eletrocardiográfica, mesmo considerando a presença de agravos cardiovasculares nesse público.

Além disso, os dados mostraram a presença de homens que adentraram a unidade e não foram submetidos às terapêuticas médicas e de Enfermagem, o que sugere que as demandas tenham sido solucionadas durante a consulta ou estiveram relacionadas a fatores, como baixa complexidade dos casos apresentados e/ou emissão de atestados médicos. Uma importante alternativa a ser repensada é a necessidade da implementação do acolhimento à demanda espontânea a ser realizada nas UBS dos municípios, como forma de conferir resolubilidade às situações clínicas de urgência.

Outro aspecto relevante evidenciado no estudo é a não identificação de agravos pré-existentes dentre às condições clínicas apresentadas pelos homens. Diante disso, é possível inferir possíveis fragilidades durante o atendimento e a triagem clínica, que podem expressar-se no insatisfatório processo de anamnese e história clínica, no desempenho das habilidades e competências profissionais para identificação desses agravos, além das problemáticas existentes nos processos de trabalho da equipe multiprofissional, em razão da elevação dos fluxos de atendimento, superlotação e não manifestação dos comportamentos, hábitos e práticas de saúde por parte dos homens.

Como um reflexo dos dados apresentados acerca dos principais diagnósticos médicos 
levantados durante os atendimentos, além da dor, os homens apresentaram manifestações clínicas relacionadas às doenças do aparelho circulatório, bem como sinais, sintomas e afecções mal definidas somadas às lesões, envenenamentos e demais causas externas e doenças osteomusculares e do tecido conjuntivo, que sinalizam a ocorrência de demandas evitáveis. Tais agravos mostram-se em convergência com o panorama nacional sobre a morbimortalidade masculina, expressando-se como um reflexo desse cenário no cotidiano dos serviços, como identifica estudo ecológico realizado em Cuiabá, Mato Grosso, Brasil, que analisou 6.050 registros de óbitos de homens ${ }^{(21)}$.

Nesse sentido, ações preventivas devem ser reforçadas, além de investimento na assistência nessas unidades, que incluam a formação de recursos humanos, para tornar os atendimentos prestados de alta qualidade e resolubilidade, com base na estruturação de linhas específicas de cuidados. Para tanto, estudo reflexivo realizado por pesquisadores do Departamento de Estudos sobre a Violência e Saúde, da Fiocruz, no Rio de Janeiro, chama a atenção para a necessidade da institucionalização da temática da violência no âmbito do SUS. Destaca-se a necessidade de superar os desafios existentes na implementação da Política Nacional de Redução da Morbimortalidade por Acidentes e Violência promulgada pelo Ministério da Saúde brasileiro ${ }^{(15)}$.

Além dos diagnósticos médicos, buscou-se investigar a existência do levantamento de Diagnósticos de Enfermagem, que se expressam de modo singular e essencial no processo de raciocínio clínico, pensamento crítico e raciocínio diagnóstico, para a tomada de decisão assistencial. No entanto, verificou-se elevada ausência de descrição desses diagnósticos pelas enfermeiras que atuam na UPA. Estudo brasileiro, realizado em um hospital universitário paulista, que buscou investigar os Diagnósticos de Enfermagem mais utilizados em serviço de emergência, recomenda o levantamento de diagnósticos para facilitar a condução da assistência de Enfermagem nesse nível de atenção à saúde e subsidiar a construção individualizada de planos de cuidados específicos para pacientes em contextos críticos de saúde ${ }^{(22-23)}$. No entanto, os achados deste estudo apontaram para a fragilidade do levantamento e documentação dos Diagnósticos de Enfermagem junto aos registros de atuação profissional das Enfermeiras ${ }^{(24)}$.

Quando analisados os desfechos apresentados na evolução dos casos, verificou-se que os homens tiveram alta por melhora, com um número reduzido de transferências decorrentes dos agravos, assim como de referência para a Atenção Básica à Saúde e com apenas um óbito no período investigado. Em articulação com a evolução do caso, o estudo permitiu identificar que os homens atendidos na UPA não eram encaminhados para outros serviços existentes na rede de atenção. Quando isso ocorria, o maior número seguia para unidades hospitalares de referência, Unidades de Saúde da Família e, em menor número, para áreas de especialidades médicas, a exemplo da cardiologia.

Esses achados exigem reflexões sobre como vêm sendo operacionalizados os atendimentos de saúde realizados pelas UPA. Importa ressaltar o fato de que os estados planejaram a existência das UPA, mas são os municípios que realizam a gestão e vêm contribuindo com o significativo número dessas unidades abertas, ainda que nem sempre funcionando plenamente. Além disso, é necessário destacar a problemática da tensão existente em relação aos hospitais, haja vista a insuficiência de leitos no país, que se alia à localização desses serviços distante das capitais dos estados, ao subfinanciamento e à dificuldade na contratação de profissionais médicos. Esses são fatores que, juntos, concorrem para que as UPA funcionem também como locais de internação ${ }^{(25)}$.

Mesmo considerando a essencialidade das UPA no alcance da resolubilidade das demandas de urgência e emergência apresentadas nos territórios, são observados distintos problemas nos municípios. Destacam-se, entre esses, a integral participação das Upa junto à Rede de Atenção à Saúde, bem como dos fluxos de atendimento, entraves no seguimento de protocolos, déficit de profissionais capacitados, dificuldades estruturais de referência e contrarreferência de pacientes na 
macrorregião de saúde e a participação na esfera estadual. Os estudos indicam ainda necessidade de fortalecimento da elaboração de sistemas informatizados e padronização de estruturas de avaliação, para que haja redução da demanda, facilitação dos fluxos assistenciais e do planejamento organizacional, para garantir o direito de acesso à saúde $e^{(13-25)}$.

De maneira também preocupante, destaca-se a ausência, nos prontuários pesquisados, das características sociodemográficas (raça/cor, escolaridade, estado civil, situação econômica) do público atendido. Este fato desvela a não importância e insensibilização para com essas variáveis, que auxiliam de maneira indispensável na formulação de indicadores em saúde que subsidiam a elaboração e implementação de políticas públicas de saúde. Além disso, a inexistência dessas informações impacta diretamente no planejamento interno das ações operacionais dos serviços. Por isso, devem ser registradas e analisadas no cotidiano das práticas de cuidado em saúde.

Por reconhecer que Enfermeiras e sua equipe de enfermagem constituem a essencialidade na produção do cuidado e de tecnologias assistenciais em saúde de homens nos serviços de urgência e emergência, e que elas encontram-se diretamente envolvidas nas ações de planejamento, supervisão, atendimento, coordenação, vigilância, gestão, monitoramento, pesquisa, avaliação e formação, este estudo é relevante para o avanço da produção do conhecimento, ao se propor a levantar evidências de que essas profissionais mostram-se capazes para a qualificação e garantia da segurança na assistência prestada a essa população.

Como limitação deste estudo, cita-se o fato de restringir-se à descrição do perfil clínico de homens em unidade de emergência, não permitindo estabelecer correlações que expliquem os fatores que estão associados à busca desses serviços. Além disso, há uma incipiência dos dados sociodemográficos decorrentes do sub-registro, o que limita a análise das informações referentes ao perfil dos usuários. Todavia, conhecer essa caracterização permite delinear linhas de cuidado e de atenção à saúde específicas na atenção aos agravos crônicos, bem como auxiliar o desenvolvimento das práticas de produção do cuidado direcionado à saúde de homens.

\section{Conclusão}

O perfil clínico de homens que frequentavam a Unidade de Pronto Atendimento 24 horas, foi caracterizado pela queixa principal de dor, com situação clínica estável, de classificação de risco considerada não urgente, sem apresentação de agravos pré-existentes. O diagnóstico médico mais prevalente foi o de dor, sendo necessária a instituição de uma terapêutica medicamentosa, com apresentação reduzida de levantamento de diagnósticos de Enfermagem.

Enquanto desfecho, os casos evoluíram para alta por melhora, não havendo encaminhamentos para outros serviços da rede de atenção à saúde. Entretanto, os achados não permitem evidenciar se há ausência de articulação ou inexistência de uma rede de atenção disponível para a saúde de homens em situação de urgência e emergência. Assim, emerge a necessidade de continuarem as investigações sobre a problemática.

Notou-se que não havia descrição dos Diagnósticos de Enfermagem nos registros de atendimento prestado por enfermeiras, o que evidencia o não cumprimento efetivo do Processo de Enfermagem. Dessa maneira, destaca-se a relevância desse registro no âmbito do cuidado de Enfermagem de emergência, para garantir a aplicação do método de trabalho em Enfermagem no cuidado prestado às pessoas, suas famílias e comunidades. Sendo assim, apontar esses achados é essencial para a gestão das práticas de cuidado, de modo que auxilie na organização dos serviços de urgência e emergência por meio da reestruturação de fluxos assistenciais, protocolos e diretrizes clínicas eficazes, quer sejam médicas, quer sejam de Enfermagem.

A descrição rigorosa dos dados de atendimento possibilita a estruturação de indicadores de qualidade da assistência prestada, assim como possibilita a instrumentalização de ações nos serviços de saúde e a implementação de 
políticas públicas, a exemplo da Política Nacional de Atenção às Urgências e Emergências e da Política Nacional de Atenção Integral à Saúde do Homem. Nesse sentido, conhecer as características clínicas desse público, permitirá delinear linhas de atenção focalizadas na rede, além de propiciar o avanço das práticas para a garantia da segurança.

\section{Colaborações:}

1 - concepção, projeto, análise e interpretação dos dados: Jéssica Cerqueira Silva, Silvia de Sousa Nassif e Anderson Reis de Sousa;

2 - redação do artigo e revisão crítica relevante do conteúdo intelectual: Selton Diniz dos Santos e Tilson Nunes Mota;

3 - aprovação final da versão a ser publicada: Anderson Reis de Sousa e Álvaro Pereira.

\section{Referências}

1. Brasil. Ministério da Saúde. Política Nacional de Atenção Integral à Saúde do homem. Brasília (DF); 2009.

2. Thompson T, Mitchell JA, Johnson-Lawrence V, Watkins DC, Modlin CS. Self-Rated Health and Health Care Access Associated with African American Men's Health Self-Efficacy. Am J Mens Health. 2017;11(5):1385-7. DOI: 10.1177/ 155798831559855

3. Baker P. Review of the national men's health policy and action plan 2008-2013: Final report for the health service executive [Internet]. Dublin (IRL): MHFI; 2015 Mar (cited 2019 Sep 10]. Available from: https://www.mhfi.org/policyreview2015. pdf

4. Sousa AR, Queiroz AM, Florencio RMS, Portela PP, Fernandes JD, Pereira A. Homens nos serviços de atenção básica à saúde: repercussões da construção social das masculinidades. Rev baiana enferm. 2016 jul/set;30(3):1-10. DOI 10.18471/rbe. v30i3.16054

5. Stockings E, Hall WD, Lynskey M, Morley KI, Reavley N, Strang J, et al. Prevention, early intervention, harm reduction, and treatment of substance use in young people. Lancet Psychiatry. 2016 Mar;3(3):280-96. DOI: 10.1016/ S2215-0366(16)00002-X
6. Mróz LW, Oliffe JL, Davison BJ. Masculinities and Patient Perspectives of Communication About Active Surveillance for Prostate Cancer. Health Psychol. 2013 Jan;32(1):83-90. DOI: 10.1037/a0029934

7. Connell RW, Messerschmidt JW. Masculinidade hegemônica: repensando O conceito. Rev Estud Fem. 2013;21(1):241-82. DOI: 10.1590/ S0104-026X2013000100014

8. Sousa AR, Pereira RM, Anjos MSB, Cerqueira AS, Alencar DC, Santana TS, et al. Acesso à saúde pela média complexidade: discurso coletivo de homens. Rev enferm UFPE on line. 2019;13:e237677. DOI: https://doi.org/10.5205/1981-8963.2019.237677

9. Elder K, Griffith DM. Men's Health: Beyond Masculinity. Am J Public Health. 2016;106(7):1157. DOI: 10.2105/AJPH.2016.303237

10. Teixeira DBS, Cruz SPL. Atenção à saúde do homem: análise da sua resistência na procura dos serviços de saúde. Rev Cubana Enfermería [Internet]. 2016 [cited 2019 Nov 25];(32):4. Available from: http:// www.revenfermeria.sld.cu/index.php/enf/article/ view/985/209

11. Feijó VBR, Cordoni Junior L, Souza RKT, Dias AO. Análise da demanda atendida em unidade de urgência com classificação de risco. Saúde Debate. 2015;39(106):627-36. DOI: 10.1590/0103-110420151060003005

12. Richardson N. Building Momentum, Gaining Traction: Ireland's National Men's Health Policy - 5 years on. New Male Studies [Internet]. 2013 [cited 2019 Sep 14];2(3):93-103. Available from:http://www.newmalestudies.com/OJS/index. $\mathrm{php/nms/article/download/90/93/}$

13. Uchimura LYT, Viana AL d'Á, Silva HP, Ibañez N. Unidades de Pronto Atendimento (UPAs): características da gestão às redes de atenção no Paraná. Saúde debate. 2015;39(107):972-83. DOI: 10.1590/0103-110420151070253

14. Philips H, Remmen R, De Paepe P, Buylaert W, Van Royen P. Out of hours care: a profile analysis of patients attending the emergency department and the general practitioner on call. BMC Fam Pract. 2010 Nov;11:88. DOI: 10.1186/1471-2296-11-88

15. Minayo MCS, Souza ER, Silva MMA, Assis SG. Institucionalização do tema da violência no SUS: avanços e desafios. Ciênc Saúde Coletiva. 2018;23(6):2007-16.DOI:10.1590/1413-81232018236. 04962018 
16. Instituto Brasileiro de Geografia e Estatística. Coordenação de Trabalho e Rendimento. Pesquisa nacional de saúde: 2013: acesso e utilização dos serviços de saúde, acidentes e violências: Brasil, grandes regiões e unidades da federação [Internet]. Rio de Janeiro; 2015 [cited 2019 Sep 20]. Available from: https://biblioteca.ibge.gov.br/visualizacao/ livros/liv94074.pdf

17. Ruane L, H Greenslade J, Parsonage W, Hawkins T, Hammett C, Lam CS, et al. Differences in Presentation, Management and Outcomes in Women and Men Presenting to an Emergency Department With Possible Cardiac Chest Pain. Heart Lung Circ. 2017 Dec;26(12):1282-90. DOI: 10.1016/j.hlc.2017.01.003

18. Salgado PO, Gonçales PC, Dantas RB, Castro MA, Chianca TCM. Diagnósticos de enfermagem em pacientes numa unidade de emergência. Rev enferm UFPE on line. 2013 jan;7(1):83-9. DOI: 10.5205/r euol.3049-24704-1-LE.0701201312

19. Guedes HM, Souza KM, Lima PO, Martins JCA, Chianca TCM. Relação entre queixas apresentadas por pacientes na urgência e o desfecho final. Rev Latino-Am Enfermagem. 2015;23(4):587-94. DOI: $10.1590 / 0104-1169.0227 .2592$

20. Acosta AM, Lima MADS. Características de usuários frequentes de serviços de urgência: revisão integrativa. Rev Eletr Enf. 2013;15(2):564-73. DOI: 10.5216/ree.v15i2.17526
21. Oliveira JCAX, Correa ACP, Silva LA, Mozer IT, Medeiros RMK. Perfil epidemiológico da mortalidade masculina: contribuições para enfermagem. Cogitare Enferm. 2017;(22)2:e49724. DOI: $10.5380 /$ ce.v22i2. 49742

22. Barreto MS, Arruda GO, Marcon SS. How adult men use and evaluate health services. Rev Eletr Enf. 2015;17(3). DOI: 10.5216/ree.v17i3.29622

23. Viveiros WL, Okuno MFP, Campanharo CRV, Lopes MCBT, Oliveira GN, Batista REA. Pain in emergency units: correlation with risk classification categories. Rev Latino-Am Enfermagem. 2018;26:e3070. DOI: 10.1590/1518-8345. 2415.3070

24. Okuno MFP, Costa N, Lopes MCBT, Campanharo CRV, Batista REA. Diagnósticos de enfermagem mais utilizados em serviço de emergência. Cogitare Enferm. 2015;20(2):385-91. DOI: 10.5380/ ce.v20i2.38606

25. O’Dwyer G, Konder MT, Reciputti LP, Lopes MGM, Agostinho DF, Alves GF. O processo de implantação das unidades de pronto atendimento no Brasil. Rev Saude Publica. 2017;51(125):1-12. DOI: https://doi. org/10.11606/S1518-8787.2017051000072

Recebido: 9 de outubro de 2019

Aprovado: 24 de outubro de 2019

Publicado: 29 de abril de 2020

A Revista Baiana de Enfermagem utiliza a Licença Creative Commons - Atribuição-NãoComercial 4.0 Internacional. https://creativecommons.org/licenses/by-nc/4.0/

Este artigo é de acesso aberto distribuído sob os termos da Licença Creative Commons (CC BY-NC). Esta licença permite que outros remixem, adaptem e criem a partir do seu trabalho para fins não comerciais. Embora os novos trabalhos tenham de lhe atribuir o devido crédito e não possam ser usados para fins comerciais, os usuários não têm de licenciar esses trabalhos derivados sob os mesmos termos. 